\title{
Interplay between the repulsive and attractive interaction and the spacial dimensionality of an excess electron in a simple fluid
}

\author{
Ashok Sethia and Eric R. Bittner \\ Department of Chemistry and Center for Materials Chemistry, \\ University of Houston, Houston, TX 77204 \\ Fumio Hirata \\ Department of Theoretical Studies, Institute for Molecular Science, \\ Myodaiji, Okazaki 444-8585, Japan
}

(Dated: December 29, 2018)

\begin{abstract}
The behavior of an excess electron in a one, two and three dimensional classical liquid has been studied with the aid of Chandler, Singh and Richardson (CSR) theory [J. Chem. Phys. 81 1975 (1984)] . The size or dispersion of the wavepacket associated with the solvated electron is very sensitive to the interaction between the electron and fluid atoms, and exhibits complicated behavior in its density dependence. The behavior is interpreted in terms of an interplay among four causes: the excluded volume effect due to solvent, the pair attractive interaction between the electron and a solvent atom, the thermal wavelength of the electron $\left(\lambda_{e}\right)$, a balance of the attractive interactions from different solvent atoms and the range of repulsive interaction between electron and solvent atom. Electron self-trapping behavior in all the dimensions has been studied for the same solvent-solvent and electron-solvent interaction potential and the results are presented for the same parameter in every dimension to show the comparison between the various dimensions.
\end{abstract}




\section{INTRODUCTION}

The behavior of an excess eletron in a wide variety of fluids has been an interesting topic

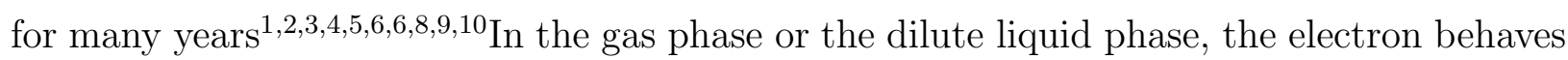
almost like a free particle. As the solvent density increases, the electron exihibits different properties depending upon the nature of the solvent and electron-solvent interactions. At liquid density, the electron may become self-trapped in a cavity of solvent particles or remain quasifree depending upon the nature of electron-solvent interaction. Observed properties

such as the electron mobility 11 and the absorption spectra 12 probe the nature of the electronic states in the fluid and phenomena of localization 14 .

There are a broad range of problems in condensed matter physics that are intimately related to the problem of excess electrons in deformable medium. These include charge transfer kinetics in biological reactions, metal-insulator transitions in fluids, polarons, phononassisted hopping of charge carriers in semiconductors and insulators, quantum-tunneling, etc. While the excess electron problem belongs to the general problem of electrons in disordered materials, the liquid environment is in many ways different from the solid medium. In liquids, the constituent particles can diffuse, and local environment around the solute electron can be substantially different from that in solid.

When an electron is solvated in a polar liquid such as water or ammonia, the strong anisotropic electron-solvent interaction causes significant local modification of the equilibrium fluid structurel 1 ? ? The electron becomes localized in a small cavity because molecules in a solvation shell orient to create a potential minimum. Even simple fluids are found to exhibit electron mobilities that change by many orders of magnitude as the density of the fluid is altered slightly. In super critical helium, for example, the electron mobility drops by over 4 orders of magnitude as fluid density increased by a factor of 2 in low density regime The reason for this behavior is strong repulsion between electron and solvent atom. This causes the the depletion of the solvent atoms from the region of the electron and forms a highly localized state of the electron.

In many other nonpolar fluids such as $\mathrm{Ar}, \mathrm{CH}_{4}$, etc. the electron always remains in a state of high mobility 15 comparable to many semiconducting materials. An interesting density dependence of the mobility has been observed in them. It shows a minimum near critical fluid density and a maximum at liquid density. 
Electronic states in reduced dimension are of considerable interest. For example, for a system less than two spatial dimension, electrons are localized with an infinitesimal amount of disorder 16. The interest in problems of electron or phonon propagation in a one dimensional random potential stems from the discovery and extensive experimental study of a certain class of organic or metallo-organic materials 17 . These matarials exhibit strongly anisotropic, quasi-one-dimensional behavior attributed to the fact that they consist of long chains, weakly interacting with each other. In many of these, the presense of a random potential has been proposed in order to explain their behavior. Electronic surface states play an important role in a wide variety of physical problems. For example, surface electrons on liquid helium has shown many interesting properties and led to important theoretical advances such as the spectrum of bound electronic states, electron transport on the He surface, effects due to deformation of the He surface, and the possibility that the electrons may form a two-dimensional crysta 18 in the field of low-dimensionality physics.

The theory for the excess electrons in fluids developed by Chandler, Singh, and Richardson (CSR) is based on the path integral formulation of quantum theory which maps the behavior of the electron on to that of a classical isomorphic polymer 19 . The solventinduced potential surface for the self-interaction of the isomorphic polymer is evaluated using an integral equation (e.g., reference interaction site model). With known potential surface, the polymer statistics is solved using variational approachen that allows the determination of electronic properties and the structure of the liquid near the electron. The input of the theory is the pure solvent structure factor and the electron-solvent particle interaction potential. The CSR theory in its formulation is applicable to an adiabatic solvent (i.e., solvent particles are treated classically), but has been extended to treat the effect of the quantum mechanical charge density fluctuation in the solvent particles21. The calculated electron-absorption line shape and mobility are in good agreement with the simulations? and experiment\$. The predictions of CSR theory were verified by computer simulation 23.24.

For a one-dimensional system w 25 have shown recently that the repulsive and attractive parts of electron-solvent interaction potential lead separately to localization of electron, respectively, by creating cavity or forming a cluster of the fluids atoms around it. In two dimensional system we 26 have shown that dispersion of the wavepacket associated with the solvated electron is very sensitive to the interaction between the electron and the fluid atoms, and exhibits complicated behavior in its density dependence. CSR theory has been extended 
to calculate the effective mass 27 as well as the density matrix of the excess electron in fluid 28 .

The CSR theory involves three or more characteristic lengths, depending upon the nature of electron-solvent interaction. These lengths are the thermal wavelength of excess electron $\lambda_{e}=\left(\frac{\beta \hbar^{2}}{m}\right)^{\frac{1}{2}}$ (where $\beta$ is the inverse of temperature in the unit of the Boltzmann constant $k_{B}$, $m$ is the mass of a bare electron, and $\hbar$ is the Planck's constant divided by $2 \pi$ ), characteristic length associated with the electron-solvent pseudopotential, and a length associated with the mean volume occupied by each solvent atom, which is related to $\rho^{*^{-\frac{1}{D}}}$, where $\rho^{*}=\rho_{s} \sigma^{D}, \rho_{s}$ being the number density of the solvent and $\sigma$ is the diameter of the solvent atom, and $D$ is the spatial dimensionality of the system. The behavior of the excess electron is expected to depend sensitively on these lengths. Laria and Chandler 29 have attempted to explain the contrasting behaviors of the electron in super critical helium and xenon on the basis of different ranges of the electron-solvent repulsive interactions.

In the present work we examine in detail the role played by different lengths and the spatial dimensionality of the system (in which we have considered the same solvent-solvent and electron-solvent model potential) to study the self-trapping behavior of the electron. The organization of the rest of the paper is as follows. In Sec. II we briefly review the CSR theory. In Sec. III we have presented the results and their discussions. Finally, Section IV presents concluding remarks. Appendix A provide some mathematical material for $D$ dimensional integration

\section{THEORY}

The system we consider is a single electron dissolved in a single component classical solvent. In the CSR theory, an excess electron is mapped, using a discretized version of the path integral formulation of quantum mechanics, onto a polymer of $\mathrm{P}$ interaction sites or

beads. Under this isomorphism 19 , the electron can be viewed as a classical ring polymer.

The total potential energy can be written as

$$
U=U_{e s}\left(\mathbf{r},\left\{\mathbf{R}_{i}\right\}\right)+U_{s s}\left(\left\{\mathbf{R}_{i}\right\}\right)
$$

with

$$
U_{s s}\left(\left\{\mathbf{R}_{i}\right\}\right)=\sum_{i>j=1}^{N} u_{s s}\left(\left|\mathbf{R}_{i}-\mathbf{R}_{j}\right|\right)
$$


and

$$
U_{e s}\left(\mathbf{r},\left\{\mathbf{R}_{i}\right\}\right)=\sum_{i=1}^{N} u_{e s}\left(\left|\mathbf{r}-\mathbf{R}_{i}\right|\right)
$$

Here, $\mathbf{r}$ denotes the position of the excess electron, $\mathbf{R}_{i}$ is the collection of the coordinates for a solvent atom, and $\mathrm{N}$ is the number of solvent atoms and $u_{e s}(r)$ and $u_{s s}(r)$ are, respectively, electron-solvent atom and solvent atom-solvent atom interaction potentian. We consider a $D$-dimensional system of spheres of diameter $\sigma$ in which the pair interaction between the solvent atoms is taken to be

$$
u_{s s}(|\mathbf{r}|)= \begin{cases}\infty & \text { for }|\mathbf{r}| \leq \sigma \\ 0 & \text { for }|\mathbf{r}|>\sigma\end{cases}
$$

where $|\mathbf{r}|$ is the $D$-dimensional distance (for notational convenience the $D$ dependence will not always be explicitly indicated). The electron-solvent atom interaction in a real system consists of a strong repulsion at short distance due to orthogonality requirements between wavefunctions of core electrons in the solvent particle and that of the excess electron and attraction at large distances due to dispersion interaction. However, in a system of neutral atoms the electronic states are determined primarily by the short range repulsive interaction or excluded-volume effect. The attractive interaction becomes important only at low densities. The interaction between the electron and solvent atom is taken to be

$$
u_{e s}(|\mathbf{r}|)= \begin{cases}\infty & \text { for } r \leq d \\ -\epsilon \frac{\exp (-\alpha r)}{\alpha r} & \text { for } r>d\end{cases}
$$

Here, $d$ is the distance of closest approach between electron and solvent atom.

In CSR theoryl the partition function $\mathrm{Z}$ for an electron in a bath of classical particles is written as the functional integral

$$
Z=\int D \mathbf{r}(u) \int d\left\{\mathbf{R}_{i}\right\} \exp \left[-\frac{1}{\hbar} \int_{0}^{\beta \hbar} d u\left\{\frac{1}{2} m|\dot{\mathbf{r}}(u)|^{2}+U_{e s}\left(\mathbf{r},\left\{\mathbf{R}_{i}\right\}\right)\right\}-\beta U_{s s}\left(\mathbf{R}_{i}\right)\right]
$$

where $\mathbf{r}(\mathrm{u})$ is the electronic path in imaginary time which is periodic in time interval $0 \leq$ $u \leq \beta \hbar$, i.e., $\mathbf{r}(0)=\mathbf{r}(\beta \hbar)$. To concentrate our attention on the electron degrees of freedom, the partition function given by Eq.(6) can be written as

$$
Z=Z_{s} \int D \mathbf{r}(u) \exp \left\{-\beta S_{\circ}[\mathbf{r}(u)-\beta \Delta \mu[\mathbf{r}(u)]\}\right.
$$

where $Z_{s}$ denotes the partition function of the solvent, $\Delta \mu[\mathbf{r}(u)]$ is the excess chemical potential for the fixed electronic path,

$$
\text { with, } \quad \beta S_{\circ}[\mathbf{r}(u)]=\frac{1}{\hbar} \int_{0}^{\beta \hbar} d u \frac{1}{2} m|\dot{\mathbf{r}}(u)|^{2}
$$


and $\exp \{-\beta \Delta \mu[\mathbf{r}(u)]\}$ is called the influence functional which represents the solvent effects on the electon. In the continuum limitl,

$$
-\Delta \mu[\mathbf{r}(u)]=\rho_{s} \hat{c}_{e s}(0)+\frac{1}{2}(\beta \hbar)^{-2} \int_{0}^{\beta \hbar} d u \int_{0}^{\beta \hbar} d u^{\prime} v\left(\left|\mathbf{r}(u)-\mathbf{r}\left(u^{\prime}\right)\right|\right)
$$

where $\hat{c}_{e s}(0)$ is the $\mathrm{k}=0$ spatial Fourier transform of $c_{e s}(r)$.

$$
v\left(\left|\mathbf{r}(u)-\mathbf{r}\left(u^{\prime}\right)\right|=-\int d \mathbf{r}^{\prime} \int d \mathbf{r}^{\prime \prime} c_{e s}\left(\mathbf{r}^{\prime}, u\right) \chi_{s s}\left(\left|\mathbf{r}^{\prime}-\mathbf{r}^{\prime \prime}\right|\right) c_{e s}\left(\left|\mathbf{r}^{\prime \prime}-\mathbf{r}\right|, u^{\prime}\right)\right.
$$

Here $\mathbf{r}$ and $u$ appear as independent coordinates, $\mathbf{r}$ is the distance between two sites and $\mathrm{u}$ measures the length along the contour of the polymer, and

$$
\chi_{s s}\left(\left|\mathbf{r}-\mathbf{r}^{\prime}\right|\right)=\left\langle\delta \rho_{s}(\mathbf{r}) \delta \rho_{s}\left(\mathbf{r}^{\prime}\right)\right\rangle
$$

is the density-density correlation function of the unpurturbed bath. In Eqs. (9) and (10), $c_{e s}$ is the direct correlation function. Its value is determined from the equation 1

$$
\rho_{s} h(\mathbf{r})=\int d \mathbf{r}^{\prime} \int d \mathbf{r}^{\prime \prime} \omega\left(\left|\mathbf{r}-\mathbf{r}^{\prime}\right|\right) c_{e s}\left(\left|\mathbf{r}^{\prime}-\mathbf{r}^{\prime \prime}\right|\right) \chi_{s s}\left(\mathbf{r}^{\prime \prime}\right)
$$

where

$$
\omega\left(\left|\mathbf{r}-\mathbf{r}^{\prime}\right|\right)=(\beta \hbar)^{-1} \int d\left(u-u^{\prime}\right) \omega\left(\left|\mathbf{r}-\mathbf{r}^{\prime}\right| ; u-u^{\prime}\right)
$$

is the intrapolymer correlation function.

Eq. (12) is solved for $c_{e s}$ and $h$ using suitable closure relation 25 . Since all sites of a ring polymer on the average are equivalent, the site dependence disappears from Eq.(12) and only the zero-frequency component $\omega(|\mathbf{r}|)$ of the equilibrium response function is required in Eq.(12).

To complete the evaluation of excess chemical potential, the electronic path integral still has to be performed. Following Feynmamen and Chandler et. al. 1 the excess chemical potential for the fixed electronic path is mimicked by a Gaussian functional,

$$
-\beta \Delta \mu_{\mathrm{ref}}[\mathbf{r}(u)]=-\Gamma_{\circ}+\frac{1}{2}(\beta \hbar)^{-2} \int_{0}^{\beta \hbar} d u \int_{0}^{\beta \hbar} d u^{\prime} \Gamma\left(u-u^{\prime}\right) \times\left|\mathbf{r}(u)-\mathbf{r}\left(u^{\prime}\right)\right|^{2}
$$

where $\Gamma\left(u-u^{\prime}\right)$ is a solvent-induced force constant between different sites on the electron polymer and $\Gamma_{\circ}$ merely determines the zero of energy. The Bogoliubov inequality provides a upper bound for the excess chemical potential,

$$
\Delta \mu \leq-\beta^{-1} \ln Z_{\mathrm{ref}}+<\Delta \mu[\mathbf{r}(u)]-\Delta \mu_{\mathrm{ref}}[\mathbf{r}(u)]>_{\mathrm{ref}}
$$


Here, $Z_{\text {ref }}$ is the electronic partion function for the Gaussian reference system and $<->_{\text {ref }}$ means the average over the reference system weight determined by $S_{\circ}+\Delta \mu[\mathbf{r}(u)]$. Minimizing the right hand side of Eq.(14) provides the optimal Gaussian reference system. This procedure leads to the following equations. The correlation function for the intrapolymer correlation in k-space:

$$
\hat{\omega}(k, \tau)=\exp \left[-\frac{k^{2} R^{2}}{2 D}\right]
$$

where

$$
R^{2}(\tau)=\left\langle\left|\mathbf{r}(u)-\mathbf{r}\left(u^{\prime}\right)\right|^{2}\right\rangle=4 D \sum_{n \geq 1}^{\infty} A_{n}\left[1-\cos \left(\Omega_{n} \tau\right)\right]
$$

is the mean square displacement between two points on the electron path separated by a imaginary time increament $0 \leq u-u^{\prime} \leq \beta \hbar$ with

$$
A_{n}=\left(\beta m \Omega_{n}^{2}+\gamma_{n}\right)^{-1}
$$

where $\Omega_{n}=\frac{2 \pi n}{\beta \hbar}$, and

$$
\gamma_{n}=-(D \beta \hbar)^{-1} \int_{0}^{\beta \hbar} d u\left[1-\cos \left(\Omega_{n} u\right)\right] \int \frac{d^{D} k k^{2}}{(2 \pi)^{D}} v(k) \exp \left(-k^{2} R^{2}(u) / 2 D\right) .
$$

We solve Eq.(12) for $h$ and $c_{e s}$ using closure relation

$$
\begin{gathered}
g=0 \text { for } r \leq d \\
c_{e s}=-\beta \epsilon \frac{\exp (-\alpha r)}{\alpha r} \text { for } r>d
\end{gathered}
$$

We can express Eq.(12) and the closure (20) in the variational form

$$
\frac{\delta I_{R I S M}}{\delta c_{e s}}=0
$$

where

$$
I_{R I S M}=\rho_{s} \hat{c}_{e s}(0)+\frac{1}{2} \int \frac{d^{D} k}{(2 \pi)^{D}} \hat{c}_{e s}^{2}(k) \hat{\chi}_{s s}(k) \hat{\omega}(k)
$$

where $\hat{c}_{e s}(0)$ is the $\mathrm{k}=0$ spatial Fourier transform of $c_{e s}(r), D$ is the dimensionality of the space, $d^{D} k$ is the dimensionality dependence volume element, $u$ labels the beads in the polymer ring $(0 \leq u \leq \beta \hbar), m$ is the bare electron mass, and $v(k)=-\hat{c}_{e s}^{2}(k) \hat{\chi}_{s s}(k)$ 
is the Fourier transform of the potential between beads, which is found in Eq.(10). The information about the electron-solvent atom interation is contained in the closure relation.

In Eq.(11), $\omega(\mathbf{r})$ is the intra polymer distribution function averaged over all beads of the ring polymer. In writing Eq.(11) it has been assumed that for each polymer configuration, the solvent sees only average polymer rather than individual beads. The intra polymer distribution function $\omega(r, \tau)$ is determined in the polaron approximation $\mathbb{\emptyset} \omega(k, u)$ is the Fourier transform of $\omega(r, u)$ and is given by

$$
\hat{\omega}(k, u)=\exp \left[-k^{2} R^{2}(u) / 2 D\right]
$$

Eq.(22) is solved self-consistently 30 for a given solvent and model potential representing the electron-fluid particle interaction. This solution gives information about $v(\mathbf{r}), \omega(\mathbf{r}), R(u)$, $\gamma_{n}$ and $g(\mathrm{r})[=1+h(\mathrm{r})]$. Note that the quantity $R(\mathrm{u})$ is the root mean square (RMS) value of the displacement between two points on the electron path separated by a time increment $0 \leq u \leq \beta \hbar$. The characteristic size or breadth of the polymer is measured by $R(\beta \hbar / 2)$. This is a measure of the spread of the wave packet associated with the particle. Since in the CSR theory a periodic boundary condition, $\mathbf{r}(0)=\mathbf{r}(\beta \hbar)$, has been imposed on the path of the electron, $R(u)$ is found to be symmetric about $u=\frac{1}{2} \beta \hbar$, i.e. it starts from zero at $\mathrm{u}=0$, attain a maximum value at $u=\frac{1}{2} \beta \hbar$ and decreases for $u>\frac{\beta \hbar}{2}$ reaching zero at $u=\beta \hbar . g(r)$ gives information about the average packing of solvent particles around the electron. The variational parameter $\gamma_{n}$ measures the strength of the electron fluid coupling. Quantities such as average kinetic energy, potential energy and effective mass etc., can be expressed in terms of $\gamma_{n} 2427$ as

$$
\begin{gathered}
\langle K . E .\rangle=\frac{D}{2} k_{B} T\left[1+\frac{\gamma_{n}}{\beta m \Omega_{n}^{2}+\gamma_{n}}\right] . \\
\langle\text { P.E. }\rangle=\rho_{s} \int d \mathbf{r} u_{e s}(|\mathbf{r}|) g(|\mathbf{r}|) . \\
\frac{m}{m^{*}}=24 \sum_{n \geq 0}^{\infty}\left(4 \pi^{2} n^{2}+\gamma_{n} \lambda_{e}^{2}\right)^{-1}
\end{gathered}
$$

In this equation $m^{*}$ is the effective mass of the solvated electron. As mentioned earlier, we need two input for this theory. One is electron-solvent and another is the densitydensity correlation function of solvent which is related to the structure factor of the solvent. 
For $D$-dimensional hard sphere solvent under cocsideration the Percus-Yevic (PY) equation can be solved analytically for $D=131$ and for $D=333$. For $D=2$ excellent results of thermodynamic and structural properties have been obtained by Baus and Colot 33 . In the above $\hat{\omega}, \hat{c}_{e s}$, and $\hat{\chi}$, are the spatial Fourier transform of $\omega, c_{e s}$, and $\chi$.

\section{RESULTS AND DISCUSSIONS}

In presenting our results we mainly focus on the imaginary time correlation function

$$
R^{2}\left(\frac{1}{2} \beta \hbar\right)=\left\langle\left|\mathbf{r}\left(\frac{1}{2} \beta \hbar\right)-\mathbf{r}(0)\right|^{2}\right\rangle=4 D \sum_{n \geq 1} A_{n}(1-\cos \pi n)
$$

and the electron-solvent radial distribution function. Note that $R\left(\frac{1}{2} \beta \hbar\right)$ gives a measure of the physical size of the electron chain or the spread of the wave packet associated with the electron. For a free particle,

$$
R\left(\frac{1}{2} \beta \hbar\right)=\sqrt{D / 4} \lambda_{e}
$$

In Fig.1 we plot the reduced correlation length, $S \equiv R\left(\frac{1}{2} \beta \hbar\right) / \sqrt{D / 4} \lambda_{e}$ which is the dispersion of the wavepacket associated with the solvated electron relative to the free particle in one, two and three dimension as a function of density for $\lambda_{e}=15 \sigma, \alpha=\sigma^{-1}$ and $d / \sigma$ $=0.29$ for several values of the attractive interaction, $\beta \epsilon$. From these figures we find that when the electron-solvent interaction is solely repulsive $(\beta \epsilon=0.0)$, the electron is always gets trapped inside a solvent cage as solvent density is increased in one and two dimensions. In three dimension [see Fig. (1C)] the repulsive interaction is not strong enough (because $d / \sigma=0.29)$ to localize the electron due to ordered structure formed when the solvent density is high. When the attractive interaction between electron and solvent atom is large $(\beta \epsilon \simeq 100)$, the reduced correlation length of the electron is very small at very low solvent density and it stays almost constant upto $\rho^{*} \sim 0.5$. This behavior strongly indicates the electron is localized to a single atom irrespective of space dimensionality. As the space dimensionality increases, the range of the constant value of the solvent density decreases for example for $D=1, S$ is constant upto $\rho^{*} \simeq 0.6$. One interesting feature we have noted from Figs. $1 \mathrm{~A}, 1 \mathrm{~B}$, and $1 \mathrm{C}$ is that as the attractive interaction increases, the reduced correlation length of the electron decreases as we increase the solvent density in the low-density regime, but the correlation turns upwardto have a maximum in the middle density regime, and 
finally the electron is trapped in the high density regime. If we carefully look at Figs.1, we observe that as we increase the space dimensionality, we find these effect are pronounsed for large attractive interaction. The similar type of behavior has been observed for the other values of $d / \sigma$. When $d / \sigma$ is less than 0.29 we found the reduced correlation length of the electron decreases as we increase the attractive interaction i.e. $\beta \epsilon$ and is self trapped at lower density. Opposite trend is found for increasing $d / \sigma$.

From Figs. $1[\mathrm{~A}], 1[\mathrm{~B}]$ and $1[\mathrm{C}]$ it is clear that at the begining when we start increasing the attractive interaction $(\beta \epsilon \simeq 20)$ the wave packet associated with the electron increases then further increase of electron solvent attractive interaction i.e. when $\beta \epsilon>50.0$ the electron is localized on a single solvent atom. The reason behind the delocalized state at small vales of attractive interaction $(\beta \epsilon)$ is the cancellation between the repulsive and attractive electronsolvent interaction. In Fig. 2[A] to Fig.2[F] we plot the electron-solvent radial distribution function for one, two and three dimension at various solvent density and attractive iteraction $(\beta \epsilon)$. Figs. 2 can be explained in consistent manner with the same physical picture given for Figs. [1]. When the attractive interaction is weak, the electron pushes the solvent atoms makes space to self-trap. When the attractive interaction is very large $(\beta \epsilon \geq 100.0)$ the electron is trapped on a single atom irrespective of space dimensionality, as evidenced by a large peak in $g(r)$ [see Figs. 2[F].

In Fig. $3[\mathrm{~A}], 3[\mathrm{~B}]$, and $3[\mathrm{C}]$ we plot the reduced correlation length as a function of $\lambda_{e} / \sigma$ at $\beta \epsilon=0$ for $D=1,2$, and 3 respectively. In one and two dimension ( Fig.3[A] and Fig.3[B] ) we observed as we increase $\lambda_{e}$ the electron is trapped in the solvent cage. If we compare Fig. 3A with Fig. 3B as the space dimensionality increases, the electron is strongly trapped in the solvent cage. One interesting feature we found in Fig. $3[\mathrm{C}]$ at $\rho^{*} \sim 0.7$ there is sharp transition from delocalized to self-trapped state. In less than two dimension when there is no attractive interaction, the electron will always be in a localized state 1 but in three dimension there is always a tendendency the electron will be in delocalized state. On the other hand, as the temperature is decreased, the electron has tendency to be in the localized state. This competition between vaious length scales probably make the sharp transition around $\lambda_{e} \sim 16 \sigma$.

In Fig. [4A], [4B] and $[4 \mathrm{C}]$ we plot the reduced correlation length as a function of density $\left(\rho^{*}\right)$ for various values of $d / \sigma$. We found for low $d / \sigma$ (for example $d / \sigma=0.15$ ) the attractive interaction dominates and the electron is self-trapped in the low density regime. On the 
other hand, when $d / \sigma$ is large the self-trapping of the electron is dominated by repulsive interaction or self-trapping is by cage effect. In the case of electron in helium and xenon, the self-trapping of electron in helium and delocalized state of the electron in xenon has been explained on the basis of $d / \sigma$ value 25 when elecron-solvent interaction is repulsive i.e. $\beta \epsilon=0$.

\section{CONCLUDING REMARKS}

The CSR theory for the excess electrons in simple fluid ( consisting of spherical atoms ) has been studied in one, two, and three dimension. The detailed study led us to the following conclusions :

1. The reduced correlation length, $S$, is very sensitive to the nature of the electronfluid atom interaction, thermal wavelength of the excess electron $\left(\lambda_{e}\right)$ and a length associated with the mean volume occupied by each fluid atom which is related to

$\rho^{*^{-\frac{1}{D}}}$, where $\rho^{*}=\rho \sigma^{D}, \rho$ being the number density of the fluid atoms and $\sigma$ is the effective diameter of a fluid atom. The behavior is interpreted in terms of an interplay among the length scales noted above. When the electron-solvent attractive interaction is very large, the density dependence of the size of the electron polymer relative to the free particle is dominated essentially by the pair attractive interaction, and the electron is trapped in a single solvent atom irrespective of the dimensionality $(D)$. On the contrary, if the attractive interaction is absent, the electron is trapped in a cage formed by the solvent atoms at higher density. When the attractive interaction is low $(\beta \epsilon \sim 10.0)$ due to cancellation between repulsive and attractive interaction, the electron is delocalized irrespective of the dimensionality.

2. In the one dimensional case the reduced correlation length is almost independent of $d / \sigma$ when electron-solvent attractive interaction is absent. On the other hand, in two and three dimensions, $S$, the reduced correlation length of the electron is sensitive on the value of the $d / \sigma$.

3. In three dimension we found when $d / \sigma=0.29$ and $\beta \epsilon=0.0$, the temperature dependence of the reduced correlation length, $S$, shows a sharp transition (metal-insulator type) around $\lambda_{e} / \sigma \sim 15$ at $\rho^{*} \sim 0.7$. 
4. The long range electron-solvent attraction dominates at low density, while hard core repulsion dominates at high densities. When both interactions are present, they can counterbalance each other. Consequently, at some intermediate density, the effective electron-solvent interaction can be quite small, resulting in the electron delocalization.

In the present work we have explored the electron self-trapping in simple liquid for various space dimension $(D=1,2$, and 3 ) which have same model potential (solvent-solvent interaction potential and electron-solvent interation potential) in every space dimension. In every space dimension (i.e. $D=1,2$, and 3 ) we have in the model system considered here there is repulsive as well as attractive electron solvent interation potential which is not considered in Refs. 25, 27, and 28. We have presented the dimensionality dependent result for self trapping behavior of electron in simple liquid.

\section{Acknowledgments}

This work was supported in part by National Science Foundation, the Robert Welch Foundation, and the Institute for Molecular Science, (Japan).

\section{APPENDIX A: D-SPACE DIMENSIONAL INTEGRATION}

In $D$-space we have for any function $\mathrm{f}(\mathbf{x})$ depending only on the distance $x=|\mathbf{x}|$,

$$
\int d \mathbf{x} f(x)=S_{D} \int_{0}^{\infty} d x x^{D-1} f(x)
$$

or on $x$ and one integration angle $\theta$,

$$
\int d \mathbf{x} f(x, \theta)=S_{D-1} \int_{0}^{\infty} d x x^{D-1} \int d \theta \sin ^{D-2} \theta f(x, \theta),
$$

where $S_{D}=D V_{D}$ is the surface area of the unit sphere of volume $V_{D}=\pi^{D / 2} / \Gamma(1+$ $D / 2)$, where $\Gamma(1+z)=z$ ! is the $\Gamma$ function.

1 D. Chandler, Y. Singh, and D. M. Richardson, J. Chem. Phys. 81, 1975 (1984).

2 D. F. Coker, B. J. Berne, and D. Thirumalai, J. Chem. Phys. 86, 5689 (1987). 
3 D. F. Coker and B. J. Berne, in Excess Electrons in Dielectric Media edited by C. Farradini and J. Jay-Gerin (Chemical Rubber, Boca Raton,1991).

4 J. Jortner and A. Gaathan, Can. J. Chem. 55, 1801 (1977).

5 D. Chandler and K. Leung, Annu. Rev. Phys. Chem. 45, 557 (1994) and relatedreferences therein.

6 G. R. Freeman, Annu. Rev. Phys. Chem. 34463 (1983); R. A. Holroyd, in Radiation Chemistry: Principles and Applications, edited by X. Farhataziz and M. A. J. Rodgers ( VCH, New York, 1987); R. A. Holroyd and W. F. Schmidt, Annu. Rev. Phys. Chem. 40, 439 (1989).

7 A. L. Nichols III and D. Chandler, J. Chem. Phys. 87, 6671 (1987).

8 D. Hsu and D. Chandler, J. Chem. Phys.93, 5075 (1990).

9 S. Miura and F. Hirata, J. Phys. Chem. 98, 9649 (1994).

10 J. Schnitker, and P. J. Rossky, J. Chem. Phys. 86, 3471 (1987).

11 N. E. Cipollini and A. O. Allen, J. Chem. Phys. 67, 131 (1977); S. S. S. Huang and G. R. Freeman, ibid 68, 1355 (1978); S. S. S. Huang and G. R. Freeman, Phys. Rev. A 24, 714 (1984); F. M. Jacobson, N. Gee, and G. R. Freeman, Phys. Rev. A 34, 2329 (1986).

12 See, for example, B. Baron, P. Delhay, and P. Lugo, J. Chem. Phys. 57, 2122 (1972); L. Kevan, ibid 56,838 (1972); D. C. Walker, Can J. Chem. 55, 1987 (1977).

14 N. F. Mott and E. A. Davis,Electronic processes in Noncystaline Materials (Clarendon, Oxford, 1971).

14 P. Krebes, J. Phys. Chem. 3702 (1984); F. -Y.Jou and G.R.Freeman, ibid. 85, 629 (1981).

15 A.Jahnke, L. Meyer, and S. A. Rice, Phys. Rev. A 3, 734 (1971); S. S. Huang and G. R. Freeman, J. Chem. Phys. 68, 1355 (1978); M. A. Floriano and G. R. Freeman, J. Chem. Phys. 85, 1603 (1986).

16 E. Abrahams, P. W. Anderson, D. C. Licciardello, and T. V. Ramakrishnan, Phys. Rev. Lett. 42, 673 (1979); P. A. Lee and T. V. Ramakrishnan, Rev. Mod. Phys. 57, 287 (1985) and relatedreferences therein.

17 A. N. Bloch, R. B. Weisman, and C. M. Varma, Phys. Rev. Lett. 28, 753 (1972); P. F. Williams, and A. N. Bloch, Phys. Rev. B 10, 1097 (1974); C. Papatriantafillou, E. N. Economou, and T. P. Eggarter,ibid. 13,910 (1976); R. Johnston, and B. Kramer, Z. Phys. B63, 273 (1986).

18 M. W. Cole in E. Y. Andrei (Ed.), Two-Dimensional Electron System, Kluwer Academic Publ., 1997; M. W. Cole, Rev. Mod. Phys. 46, 451 (1974); C. C. Grimes, 73, 379 (1978); V. B. Shikin, 
73, 396 (1978).

19 D. Chandler and P. G. Wolynes, J. Chem. Phys. 74, 4078 (1981).

20 R. P. Feynman, Phys. Rev., 97, 660 (1955); Statistical Mechanics, edited by J. Shahmam (Benjamin, New York, 1972).

21 A. L. Nichols III and D. Chandler, J. Chem. Phys. 84, 398 (1986).

22 D. F. Coker and B. J. Berne, J. Chem. Phys. 892128 (1988).

23 M. Sprik, M. L. Klein, and D. Chandler, J. Chem. Phys. 83, 3042 (1985).

24 G. Malescio and M. Parrinello, Phys Rev.A 35, 897 (1987).

25 A. Sethia and Y. Singh, Phys. Rev. B42, 6090 (1990).

26 A. Sethia, Y Singh, and F. Hirata, Chem. Phys. Lett. 326, 199 (2000).

27 A. Sethia and Y. Singh, Phys. Rev. B46, 9958 (1992).

28 A. Sethia, F. Hirata and Y. Singh, J. Chem. Phys. 110, 10086 (1999); A. Sethia, S. Miura and F. Hirata, J. Mol. Liq. 90225 (2001).

29 D. Laria and D. Chandler, J. Chem. Phys. 87, 4088 (1987).

30 A. L. Nichols III,D. Chandler, Y. Singh, and D. M. Richardson, J. Chem. Phys. 81, 5109 (1984).

31 Z. W. Salsburg, R. W. Zwanzig and J. C. Kirkwood, J. Chem. Phys. 211098 1953).

32 M. S. Wertheim, Phys. Rev. Lett. 10, 321 (1963); E. Theile, J. Chem. Phys. 39, (1963).

33 M. Baus and J. L. Colot, Phys. Rev. A36, 3912 (1987). 

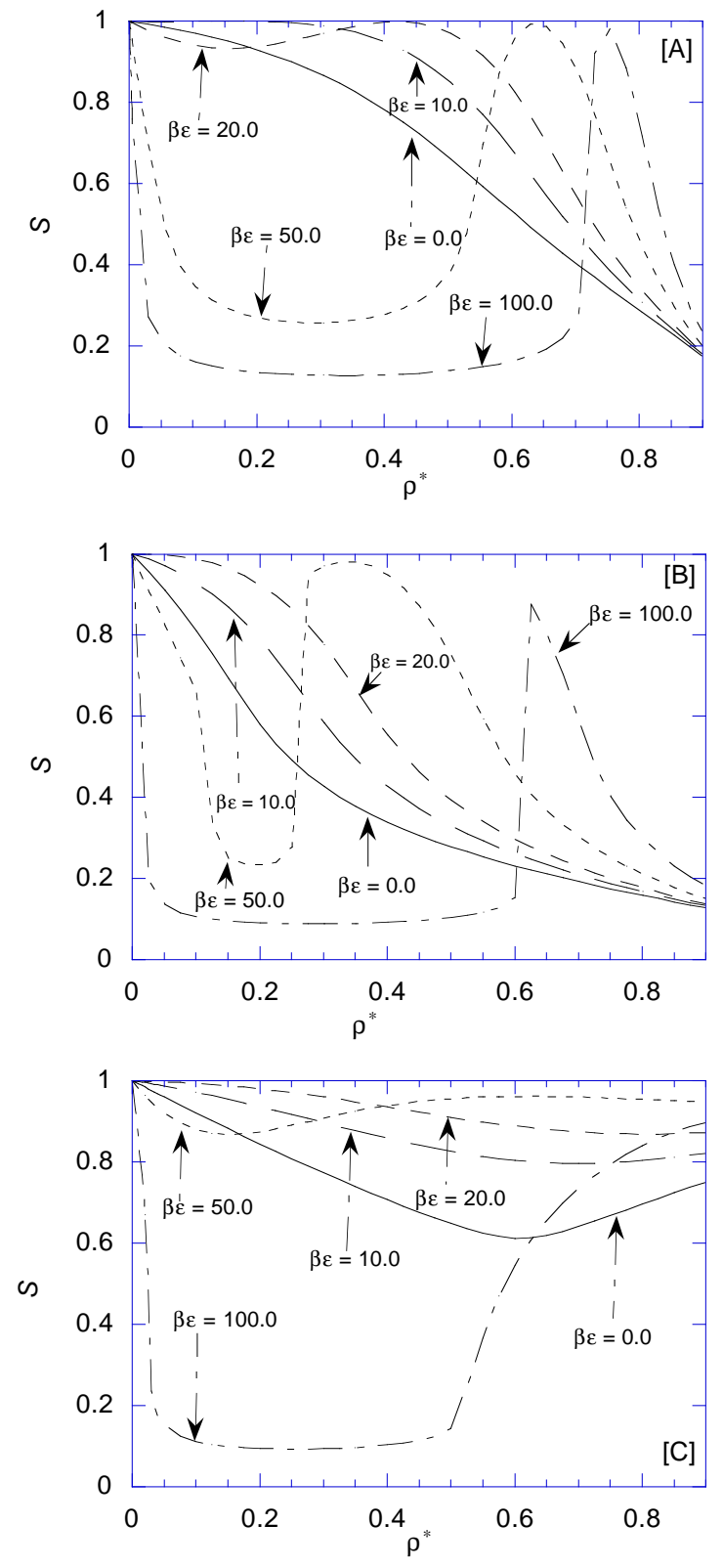

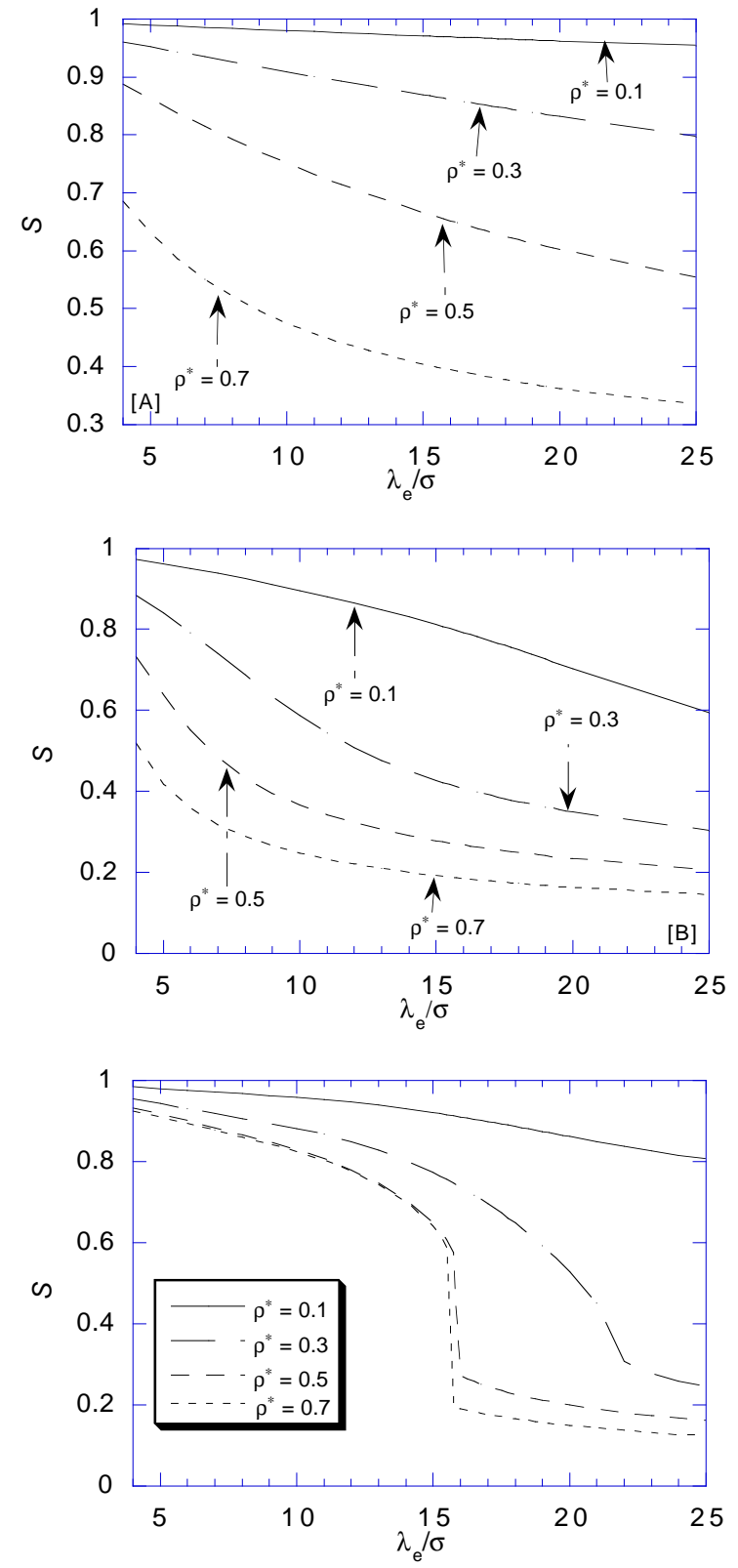

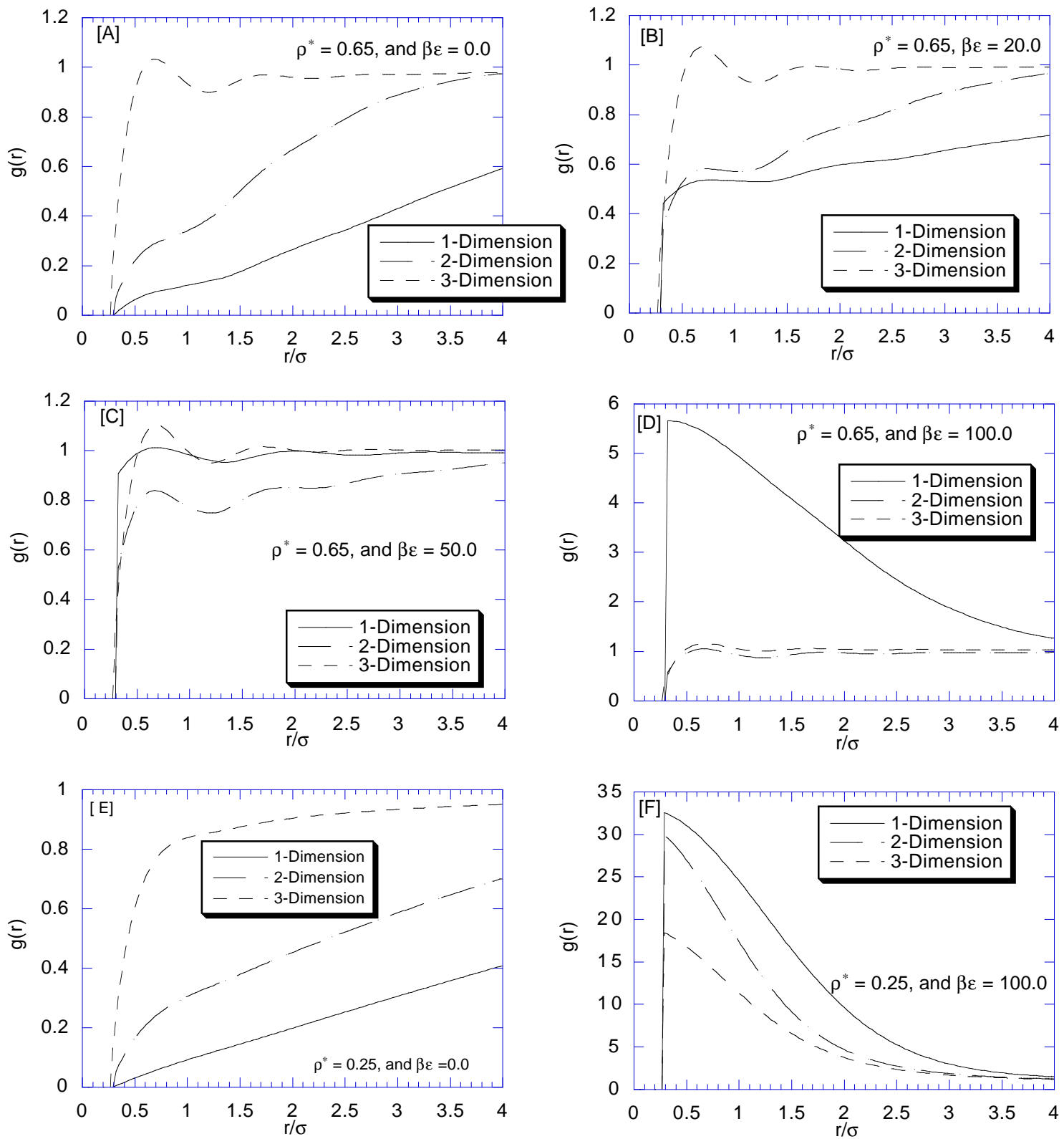

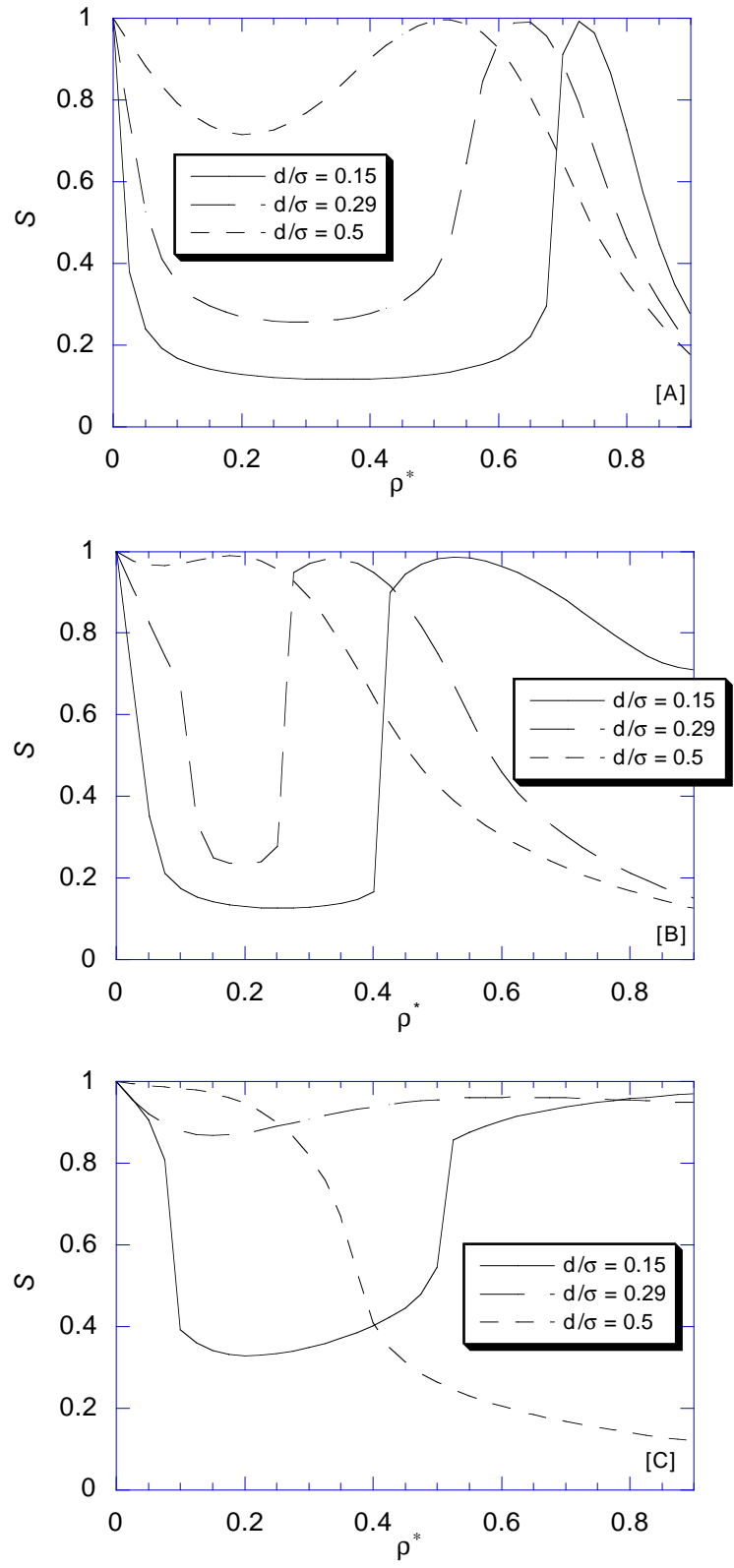